\title{
A tumorsphere model of glioblastoma multiforme with intratumoral heterogeneity for quantitative analysis of cellular migration and drug response
}

Gudbergsson, Johann Mar; Kostrikov, Serhii; Johnsen, Kasper Bendix; Fliedner, Frederikke Petrine; Stolberg, Christian Brøgger; Humle, Nanna; Hansen, Anders Elias; Kristensen, Bjarne Winther; Christiansen, Gunna; Kjær, Andreas

Total number of authors:

12

Published in:

Experimental Cell Research

Link to article, DOI:

10.1016/j.yexcr.2019.03.031

Publication date:

2019

Document Version

Peer reviewed version

Link back to DTU Orbit

Citation (APA):

Gudbergsson, J. M., Kostrikov, S., Johnsen, K. B., Fliedner, F. P., Stolberg, C. B., Humle, N., Hansen, A. E. Kristensen, B. W., Christiansen, G., Kjær, A., Andresen, T. L., \& Duroux, M. (2019). A tumorsphere model of glioblastoma multiforme with intratumoral heterogeneity for quantitative analysis of cellular migration and drug response. Experimental Cell Research, 379(1), 73-82. https://doi.org/10.1016/j.yexcr.2019.03.031

\section{General rights}

Copyright and moral rights for the publications made accessible in the public portal are retained by the authors and/or other copyright owners and it is a condition of accessing publications that users recognise and abide by the legal requirements associated with these rights.

- Users may download and print one copy of any publication from the public portal for the purpose of private study or research.

- You may not further distribute the material or use it for any profit-making activity or commercial gain

- You may freely distribute the URL identifying the publication in the public portal 


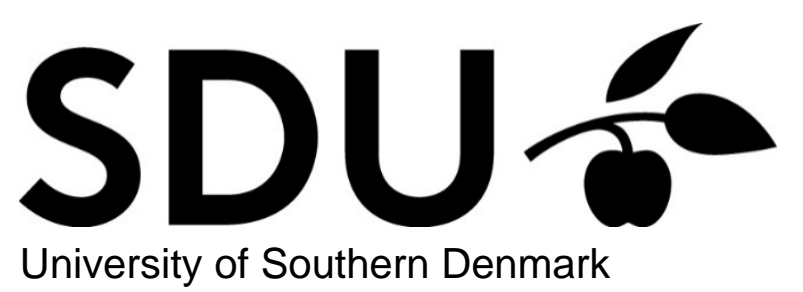

\section{A tumorsphere model of glioblastoma multiforme with intratumoral heterogeneity for quantitative analysis of cellular migration and drug response}

Gudbergsson, Johann Mar; Kostrikov, Serhii; Johnsen, Kasper Bendix; Fliedner, Frederikke Petrine; Stolberg, Christian Brøgger; Humle, Nanna; Hansen, Anders Elias; Kristensen, Bjarne Winther; Christiansen, Gunna; Kjær, Andreas; Andresen, Thomas Lars; Duroux, Meg Published in:

Experimental Cell Research

\section{DOI:}

10.1016/j.yexcr.2019.03.031

Publication date:

2019

Document version

Accepted manuscript

Document license

CC BY-NC-ND

Citation for pulished version (APA):

Gudbergsson, J. M., Kostrikov, S., Johnsen, K. B., Fliedner, F. P., Stolberg, C. B., Humle, N., Hansen, A. E., Kristensen, B. W., Christiansen, G., Kjær, A., Andresen, T. L., \& Duroux, M. (2019). A tumorsphere model of glioblastoma multiforme with intratumoral heterogeneity for quantitative analysis of cellular migration and drug response. Experimental Cell Research, 379(1), 73-82. https://doi.org/10.1016/j.yexcr.2019.03.031

\section{Terms of use}

This work is brought to you by the University of Southern Denmark through the SDU Research Portal.

Unless otherwise specified it has been shared according to the terms for self-archiving.

If no other license is stated, these terms apply:

- You may download this work for personal use only.

- You may not further distribute the material or use it for any profit-making activity or commercial gain

- You may freely distribute the URL identifying this open access version 


\section{Accepted Manuscript}

A tumorsphere model of glioblastoma multiforme with intratumoral heterogeneity for quantitative analysis of cellular migration and drug response

Johann Mar Gudbergsson, Serhii Kostrikov, Kasper Bendix Johnsen, Frederikke Petrine Fliedner, Christian Brøgger Stolberg, Nanna Humle, Anders Elias Hansen, Bjarne Winther Kristensen, Gunna Christiansen, Andreas Kjær, Thomas Lars Andresen, Meg Duroux

PII: S0014-4827(19)30131-4

DOI: https://doi.org/10.1016/j.yexcr.2019.03.031

Reference: YEXCR 11368

To appear in: Experimental Cell Research

Received Date: 27 November 2018

Revised Date: 20 March 2019

Accepted Date: 22 March 2019

Please cite this article as: J.M. Gudbergsson, S. Kostrikov, K.B. Johnsen, F.P. Fliedner, Christian.Brø. Stolberg, N. Humle, A.E. Hansen, B.W. Kristensen, G. Christiansen, A. Kjær, T.L. Andresen, M. Duroux, A tumorsphere model of glioblastoma multiforme with intratumoral heterogeneity for quantitative analysis of cellular migration and drug response, Experimental Cell Research (2019), doi: https://doi.org/10.1016/ j.yexcr.2019.03.031.

This is a PDF file of an unedited manuscript that has been accepted for publication. As a service to our customers we are providing this early version of the manuscript. The manuscript will undergo copyediting, typesetting, and review of the resulting proof before it is published in its final form. Please note that during the production process errors may be discovered which could affect the content, and all legal disclaimers that apply to the journal pertain. 
1 Title: A tumorsphere model of glioblastoma multiforme with intratumoral heterogeneity for quantitative

2 analysis of cellular migration and drug response

3 Running title: Intratumoral heterogeneity in a glioblastoma model

4 Johann Mar Gudbergsson ${ }^{1 *}$, Serhii Kostrikov ${ }^{2}$, Kasper Bendix Johnsen ${ }^{2}$, Frederikke Petrine Fliedner ${ }^{3}$,

5 Christian Brøgger Stolberg ${ }^{1}$, Nanna Humle ${ }^{4}$, Anders Elias Hansen ${ }^{2}$, Bjarne Winther Kristensen $^{5,6}$, Gunna

6 Christiansen ${ }^{7}$, Andreas Kjær $^{3}$, Thomas Lars Andresen ${ }^{2}$, Meg Duroux $^{1 *}$

$7{ }^{1}$ Laboratory of Immunology and Cancer Biology, ${ }^{4}$ Laboratory of Neurobiology, Institute of Health Science

8 and Technology, Aalborg University, Aalborg, Denmark, ${ }^{2}$ Center for Nanomedicine and Theranostics,

9 Department of Health Technology, Technical University of Denmark, Lyngby, Denmark, ${ }^{3}$ Cluster for

10 Molecular Imaging, Department for Biomedical Sciences \& Department of Clinical Physiology, Nuclear

11 Medicine \& PET, Rigshospitalet, University of Copenhagen, Copenhagen, Denmark, ${ }^{5}$ Department of

12 Pathology, Odense University Hospital, Odense, Denmark, ${ }^{6}$ Department of Clinical Research, University of

13 Southern Denmark, Odense, Denmark, ${ }^{7}$ Department of Biomedicine, Aarhus University, Aarhus, Denmark.

$14 *$ Address correspondence to

15 Johann Mar Gudbergsson, M.Sc.

16 Laboratory of Immunology and Cancer Biology, Department of Health Science and Technology, Aalborg

17 University

18 Fredrik Bajers Vej 3B, 9220 Aalborg $\varnothing$, Denmark

19 E-mail: jmg@hst.aau.dk

$20 *$ Address correspondence to

21 Meg Duroux, Ph.D.

22 Laboratory of Immunology and Cancer Biology, Department of Health Science and Technology, Aalborg 
University

Fredrik Bajers Vej 3B, 9220 Aalborg $\varnothing$, Denmark

E-mail: megd@hst.aau.dk

\section{ABSTRACT}

Glioblastoma multiforme (GBM) is the most common and malignant type of primary brain tumor and is characterized by its sudden onset and invasive growth into the brain parenchyma. The invasive tumor cells evade conventional treatments and are thought to be responsible for the ubiquitous tumor regrowth. Understanding the behavior of these invasive tumor cells and their response to therapeutic agents could help improve patient outcome. In this study, we present a GBM tumorsphere migration model with high biological complexity to study migrating GBM cells in a quantitative and qualitative manner. We demonstrated that the in vitro migration model could be used to investigate both inhibition and stimulation of cell migration with oxaliplatin and GBM-derived extracellular vesicles, respectively. The intercellular heterogeneity within the GBM tumorspheres was examined by immunofluorescent staining of nestin/vimentin and GFAP, which showed nestin and vimentin being highly expressed in the periphery of tumorspheres and GFAP mostly in cells in the tumorsphere core. We further showed that this phenotypic gradient was present in vivo after implanting dissociated GBM tumorspheres, with the cells migrating away from the tumor being nestin-positive and GFAP-negative. These results indicate that GBM tumorsphere migration models, such as the one presented here, could provide a more detailed insight into GBM cell biology and prove highly relevant as a pre-clinical platform for drug screening and assessing drug response in the treatment of GBM.

Keywords: glioblastoma; GBM; migration; invasion; nestin; GFAP; tumorsphere; extracellular vesicles; oxaliplatin 


\section{INTRODUCTION}

Glioblastoma multiforme (GBM) is the most common and aggressive primary brain tumor with a median overall survival of only 15 months with the present standard of care [1]. Current best practice for treating these tumors consists of maximal surgical resection followed by concomitant radio- and chemotherapy, but recurrence of the tumor still remains ubiquitous [2]. GBM is characterized by its rapid growth and invasion into the surrounding brain parenchyma, high vascularization, and hypoxic niches harboring cancer stem-like cells within the tumor milieu [3]. Therefore, the complexity in the study of GBM resides in the heterogenous nature at the molecular and cellular level, which hinders the derivation of representative in vitro and in vivo GBM models. The study of GBM's ability to invade the brain parenchyma could potentially reveal new targets for treatment by helping researchers understand the mechanisms driving cell invasion. To facilitate this understanding, in vitro migration or invasion assays are commonly used [4]. Identification of drugs or factors that can inhibit or stimulate cancer cell migration also rely on the use of in vitro studies to select promising candidates for further assessment in vivo.

In vitro, invasion and migration assays are typically defined by separate parameters: Invasion assays are characterized by embedding cells in a 3D milieu where a restructuring of the extracellular matrix (ECM) takes place, whereas migration is defined by cells moving on a 2D ECM, i.e. Matrigel or collagen matrices [4]. Many migration assays today rely on the use of adherent monolayer cell cultures (2D cultures) that typically are dependent on the addition of serum to the growth medium for cell propagation [4]. In recent years, more focus has been drawn to the use of cancer cell lines that are cultured as non-adherent tumorsphere cultures without the addition of serum (3D cultures) [5]. Such cells have usually been cultured in medium favoring stem-like properties that enable the formation of tumorspheres from single cancer cells. When reaching a certain size, tumorspheres can display different cellular phenotypes generating a more complex tumor-like composition [6]. The invasive potential of cancer cells has been directly correlated to their degree of malignancy, and often the cells found to facilitate the process of tissue 
invasion and metastasis have been identified as cancer cells with stem-like properties $[7,8]$. The extracellular matrix in the tissue harboring the tumor plays an important role in cancer cell invasion, modulating which cells move and which cellular pathways are utilized during the event [9]. This complex microenvironment can, to some extent, be mimicked in in vitro migration assays where different matrix components applied can affect the cells in different ways. For example, some studies apply a matrix constituted of only a single type of ECM protein such as collagens or fibronectin, and others apply more complex mixtures such as Matrigel [9-12].

In this study, we provide a more biologically relevant model with respect to cell migration by combining primary tumorsphere cell cultures and complex ECM to create a more relevant milieu with respect to cancer cell migration. We refine an established tumorsphere migration model to include both real time quantification and the possibility to do subsequent high-resolution microscopy to assess tumorsphere characteristics. The model uses a primary GBM cell line grown on Geltrex. A characterization of intra-tumorsphere cellular heterogeneity was done by visualizing a gradient in nestin/vimentin and Glial Fibrillary Acidic Protein (GFAP) expression between the tumorsphere periphery and core. The in vitro study was supported by ex vivo examination of such phenotypic gradient in an orthotopic mouse GBM xenograft generated with the same GBM tumorspheres. To illustrate that this model can be used to both inhibit and stimulate GBM cell migration, we used oxaliplatin and extracellular vesicles (EVs) derived from GBM cells, respectively, hereby underscoring its potential as an assay of therapeutic efficacy.

\section{MATERIALS AND METHODS}

\section{Ethical approval}

All experimental procedures were approved by the Danish Animal Welfare Council, the Danish Ministry of Justice (license no. 2019-15-0201-00920). NMRI nude mice (Taconic Biosciences, Denmark) were housed in 
IVC rack in Type III SPF cages with a maximum of 8 mice in each cage. Food and water were available ad libitum.

\section{Cell culture}

Primary GBM tumorsphere cultures T78 were generated as previously described and cultured in Neurobasal A medium supplemented with $1 \%$ B27 supplement, $2 \mathrm{mM} \mathrm{L-glutamine} \mathrm{and} 20 \mathrm{ng} / \mathrm{mL}$ EGF and bFGF and penicillin-streptomycin (100 U/mL penicillin and $100 \mu \mathrm{g} / \mathrm{mL}$ streptomycin) $[13,14]$. T78 cells were used at passages 18-20 throughout all experiments. For EV isolation, a secondary GBM cell line was cultured in DMEM-F12 supplemented with $10 \%$ EV-depleted FCS and penicillin-streptomycin $(100 \mathrm{U} / \mathrm{mL}$ penicillin and $100 \mu \mathrm{g} / \mathrm{mL}$ streptomycin). EV-depleted FCS was generated by ultracentrifugation of FCS at 120,000 RCF for 16 hours, where the supernatant was further used for culturing cells for EV production.

\section{Geltrex coating}

Geltrex (ThermoFisher, MA, USA; \#A1413302) was thawed on ice at $4^{\circ} \mathrm{C}$ prior to use. After thawing, Geltrex matrix was diluted 1:50 in growth medium and seeded in a volume of $700 \mu \mathrm{L}$ per well into the middle eight wells of a 24 well plate. Everything was kept cool on ice while resuspending and coating the wells. The plates were then incubated at $37^{\circ} \mathrm{C}$ for minimum 4 hours to let the Geltrex matrix solidify.

\section{Isolation of single tumorspheres and treatment}

Prior to GBM tumorsphere isolation, the plates were cooled to room temperature (usually 10-20 min), and the medium was then removed from the wells. Tumorspheres were selected according to their size (approximately $100-200 \mu \mathrm{m}$ in diameter) and isolated with a pipette in a volume of $0.5 \mu \mathrm{L}$ under a phasecontrast microscope. One tumorsphere was spotted into the middle of each well. The surrounding wells were filled with $500 \mu \mathrm{L} \mathrm{mL}$ PBS to avoid evaporation and drying of the tumorspheres. After spotting the tumorspheres, the plates were incubated at $37^{\circ} \mathrm{C}$ for $30-45 \mathrm{~min}$ to allow adherence to the gel, and then 700 $\mu \mathrm{L}$ of pre-heated growth medium was carefully added to each tumorsphere-containing well. 
The day after spotting the tumorspheres (referred to as day 1 or D1), treatment groups were randomly assigned. Tumorspheres received a single-dose of oxaliplatin on day 1 at a concentration of $5 \mu \mathrm{M}$, similar to the concentration used in other studies [15]. EVs were added in a concentration of approximately $6.5 \times 10^{7}$ particles per well in triplicates. EVs isolated from non-conditioned medium were included in triplicates as a control to account for the potential effects of EVs or other factors remaining in the medium. No treatment controls (NTC) were done in five replicates. TGF- $\beta 1$ was added to the cells in a concentration of $4 \mathrm{ng} / \mathrm{mL}$ in triplicates.

\section{Quantitative data acquisition and analysis}

Phase-contrast images were acquired each day for a total of 5 days (D0 - D4) with a Zeiss Axio Observer Z.1 (DE). Area of the growing spheres was estimated with Zeiss ZEN2 Blue Edition. All graphs were generated in GraphPad Prism 6.

\section{Fluorescence microscopy and time-lapse imaging on tumorspheres in vitro}

Sterile coverslips were placed in each well, and Geltrex coating was done as previously described. Bulk GBM tumorspheres were seeded (10-30 per well) in growth medium and incubated overnight. Tumorspheres were washed in PBS and fixed in $4 \%$ formaldehyde for $15 \mathrm{~min}$. at room temperature. Tumorspheres were then washed again and blocked in $5 \%$ BSA PBS for 30 min. Primary antibodies Ms anti-human nestin (Abcam, Cambridge, UK; \#ab22035; 1:1000), Ms anti-vimentin (Abcam; \#ab92547; 1:1000), Rb anti-GFAP (Dako, DK; \#ZO334; 1:1000), were added to cells in $0.5 \%$ BSA PBS and incubated on a rocking table overnight at $4^{\circ} \mathrm{C}$. Tumorspheres were then washed and secondary antibodies Dnk-anti-Ms-Alexa-488 (ThermoFisher; \#R37114; 1:1000), Dnk-anti-Rb-Alexa-555, (ThermoFisher; \#A-31572; 1:1000) were added to cells and incubated on a rocking table for 2 hours at room temperature. Tumorspheres where then washed and stained with Hoechst33342 (ThermoFisher; \#H3570; 1:3000) for 10 min on a rocking table at room temperature. Coverslips were transferred to SuperFrost (Menzel Gläser, ThermoFisher) slides with a drop of fluorescent mounting medium (Dako; \#S3023) and stored in a fridge at $4-6^{\circ} \mathrm{C}$ overnight to harden. 
Images were obtained on Zeiss Observer Z.1 with Apotome-2 structured illumination microscopy with a 40x NA1.30 objective (Zeiss). Quantification of GFAP:nestin ratio between core and periphery was done by threshold analyses of 4 images and presented as a bar chart with mean + SD in GraphPad Prism 6. Timelapse imaging was done on tumorspheres directly after seeding onto Geltrex and imaged on a Zeiss Observer Z.1 with a mounted Pecon Incubator P S compact (Pecon, Erbach, DE). Images were acquired with Zeiss ZEN2 Blue software every $10 \mathrm{~min}$. with automated focus over the course of 24 hours. Images and time-lapse series were processed and analyzed in Fiji [16].

\section{Paraffin embedding and immunostaining of free-floating GBM tumorspheres in vitro}

Tumorspheres were fixed free-floating in methanol for five minutes before embedding in paraffin for sectioning. $5 \mu \mathrm{m}$ sections of embedded spheroids were cut on a Leica RM 2255 microtome (Nussloch, DE) and fixated on glass slides by melting of paraffin residue at $60^{\circ} \mathrm{C}$ for one hour. Sections were stained using primary antibodies Rb anti-GFAP (Dako; \#ZO334; 1:200) and Ms anti-human nestin (Abcam; \#ab22035; 1:200). Secondary antibodies were Dnk-anti-Ms-Alexa-488 (Invitrogen; \#A-21202; 1:500) and Gt-anti-RbAlexa-594 (Invitrogen, CA, USA; \#A-11037; 1:500). Antigen retrieval was performed using a $10 \mathrm{mM}$ sodium citrate buffer $(\mathrm{pH}$ 6.0) with $0.05 \%$ Tween. Cells were additionally immunostained with 4,6-diamidino-2phenylindole (DAPI) (Sigma; \#000000010236276001; 1:500) for nuclear staining. Slides were mounted using fluorescent mounting medium (Dako; \#S3023) and images were acquired on a Zeiss Observer Z.1 using the Colibri light source (Zeiss) and Orca-Flash4.0 V2 (Hamamatsu) as the detector. To quantify the area that nestin and GFAP signal covers, the manual threshold tool in Fiji was used. Five images of five different tumorspheres were used for thresholding and the area coverage in percent was normalized for each tumorsphere to the respective tumorsphere size determined by area of the nuclear stain (DAPI) when oversaturated. Data was plotted and analyzed in GraphPad Prism 6.

\section{GBM mouse xenograft model}


163 T78 GBM tumorspheres were grown until 100-200 $\mu \mathrm{m}$ in diameter and dissociated with TrypLE 164 (ThermoFisher; \#12604013). Cells were then washed twice, counted, and cell numbers adjusted to 20.000

165 cells $/ \mu \mathrm{L}$. A total of $10 \mu \mathrm{L}$ ( 200.000 cells) was resuspended in growth medium and injected into the striatum ( $0.5 \mathrm{~mm}$ below Bregma, $1.5 \mathrm{~mm}$ lateral) of nude NMRI mice using a syringe pump running at $30 \mathrm{~nL} / \mathrm{s}$. To avoid cells being dragged back up with the removal of the needle, the needle was left in the injection site for 3 minutes prior to removal. Tumor size and growth was monitored with MRI (BioSpec 7T, Bruker, Mannheim, DE) using T2-weighted sequence of the mouse brain obtained in axial and coronal directions (Figure S1). Mice were anesthetized with Sevoflurane when the tumor size reached $10-20 \mathrm{~mm}^{3}$ and transcardially perfused with PBS followed by perfusion of $4 \%$ methanol-free paraformaldehyde. Brains were removed from the skull and post-fixed overnight at $4^{\circ} \mathrm{C}$.

\section{Fluorescence immunohistochemistry on GBM tumors}

Brains were immersed in cryoprotection with $10 \%, 20 \%$ and $30 \%$ sucrose (each step overnight), embedded in OCT (Micro and Nano; \#16-004004) and frozen in isopentane on dry ice. $30 \mu \mathrm{m}$ coronal sections were obtained with a cryostat (Leica CM 1850 UV). Sections were blocked with blocking solution containing $5 \%$ donkey serum (Millipore, Darmstadt, DE; \#S30-100ML) and 0.2 \% saponin (VWR, DK; \#27534.187) in TBS for 1 hour. The sections were then blocked with mouse on mouse blocking reagent (Vector Laboratories, CA, USA; Cat. \#MKB-2213) and after 2 hours, the solution was changed to mouse on mouse blocking reagent in $0.2 \%$ saponin in TBS for 1.5 hours. The sections were incubated with primary antibodies: Rb anti-human GFAP (Abcam; \#ab33922; 1:500) and Ms anti-human nestin (Abcam; \#ab22035; 1:400) overnight at $4^{\circ} \mathrm{C}$. After washing in TBS, sections were stained with Hoechst 33342 (ThermoFisher; \#62249; 1:1000) and secondary antibodies: Dnk-anti-Rb-Alexa-568 (Invitrogen; Cat. \#A10042; 1:1000) Dnk-anti-Ms-Alexa-647 (Invitrogen; Cat. \#A-31571; 1:1000) for 3 hours at room temperature. Sections were washed and mounted using ProLong ${ }^{\mathrm{TM}}$ Diamond Antifade Mountant mounting media (Invitrogen; \#P36970). Samples were imaged with a confocal laser scanning microscope (Zeiss LSM 710) and fluorescence slide scanner (Zeiss Axio 
Scan.Z1). For the images obtained with fluorescent slide scanning shading correction was applied using Zeiss ZEN Blue 2.3 software. Secondary antibody controls are presented in Figure S2.

\section{EV isolation}

EVs were isolated from GBM cells grown in DMEM-F12 supplemented with $10 \%$ FCS and $1 \%$ penicillinstreptomycin. To produce conditioned medium (CM), EV-depleted FCS was made by ultracentrifugation of FCS at 120,000 RCF for $>16$ hours. The EV-depleted FCS was then diluted to $10 \%$ in DMEM-F12 and added to the cells in T175 flasks $\left(30 \mathrm{~mL}\right.$ ) and incubated for 24 hours at $37^{\circ} \mathrm{C}$. CM was harvested and centrifuged for $20 \mathrm{~min}$ at $2000 \mathrm{RCF}$ and either stored at $-20^{\circ} \mathrm{C}$ until further processing (for maximum two weeks) or processed directly. The supernatant was transferred to a new tube and centrifuged at 9000 RCF for 30 min. The supernatant was filtered through $0.2 \mu \mathrm{m}$ filters and centrifuged at 120,000 RCF for 2.5 hours. The resulting supernatant was discarded, and the pellet resuspended in growth medium or Trehalose-PBS, for either Tumorsphere migration assay or NTA and TEM validation (see below), respectively. The EV CTRL was made by running non-conditioned medium through the exact same EV isolation protocol.

\section{Nanoparticle Tracking Analysis}

All NTA analyses were done on a NanoSight LM-10 (Malvern, UK). A dilution of the EVs was made to include around $50-100$ particles at once in the field of view. For video recording, shutter was between 700 and 800 , gain was between 550 and 620 , and the capture time for each recording was $30 \mathrm{~s}$. For each sample, a total of five videos were recorded. Prior to NTA, screen gain was adjusted to 2 , blur was set to $3 \times 3$, and detection threshold set between 16 and 28. Tracks were exported to Microsoft Excel and imported into GraphPad Prism 6 (GraphPad, CA, USA) for further analysis.

\section{Immunoelectron microscopy of immunogold-labelled EVs}

Immunolabelling was performed by mounting 5 uL concentrated samples on carbon- coated, glow discharged 400 mesh $\mathrm{Ni}$ grids for $30 \mathrm{~s}$ and washed 3 times with PBS. Grids were blocked with 0.5\% 
ovalbumin in PBS and then incubated with a cocktail of primary anti-CD9 (Ancell, MN, USA; \#SN4/C3-3A2; 1:50), anti-CD63 (Ancell; \#AHN16.1/46-4-5; 1:50) and anti-CD81 (Ancell; \#1.3.3.22; 1:50) monoclonal antibodies in $0.5 \%$ ovalbumin in PBS for $30 \mathrm{~min}$ at $37^{\circ} \mathrm{C}$. After incubation grids were washed 3 times with PBS and incubated with secondary antibody goat anti-mouse conjugated with $10 \mathrm{~nm}$ colloidal gold (British BioCell, Cardiff, UK) 1:25 in $0.5 \%$ ovalbumin in PBS for $30 \mathrm{~min}$ at $37^{\circ} \mathrm{C}$. The grids were then washed with 3 drops of PBS, before incubation on 3 drops of $1 \%$ cold fish gelatin for $10 \mathrm{~min}$ each. The grids were finally washed with 3 drops of PBS before staining with 1 drop of $1 \%(w / v)$ phosphotungstic acid at $\mathrm{pH} 7.0$ and blotted dry. Images were obtained with a transmission electron microscope (JEM-1010, JEOL, Eching, Germany) operated at $60 \mathrm{keV}$ coupled to an electron- sensitive CCD camera (KeenView, Olympus, Center Valley, PA, USA). For size determination of visible EVs a grid-size replica $(2,160$ lines $/ \mathrm{mm})$ was used. See Table S1 for full antibody list.

\section{Production of oxaliplatin-loaded stealth liposomes}

Stealth liposomes were produced from a lipid formulation containing hydrogenated soybean phosphatidylcholine (HSPC), DSPE-PEG 2000 , and cholesterol (Lipoid GmbH, Ludwigshafen, DE) in a molar ratio of 56.8:38.2:5 mol\%. Hydration of the lipid powder was done for 1 hour at $65^{\circ} \mathrm{C} 10 \mathrm{mM} \mathrm{HEPES}$ and $5 \%$ glucose (pH 7.4) containing oxaliplatin (Lianyungang Guiyuan Chempharm Co., LTD, Jiangsu, PRC). Extrusion of the liposomes and determination of phospholipid and oxaliplatin concentration were performed as described in Johnsen et al. (2019) [17]. The hydrodynamic diameter and $\zeta$-potential of the resulting oxaliplatin-loaded stealth liposomes were measured with a Zetasizer (ZetaPALS, Brookhaven Instruments Ltd., NY, USA), showing a diameter of approximately $120 \mathrm{~nm}$ and a net negative surface charge.

\section{RESULTS}

\section{Intra-tumorsphere cellular heterogeneity display in vitro}


Since GBM tumorspheres grow in a non-adherent 3D fashion, we hypothesized that a cellular heterogeneity could arise within each tumorsphere. First, time-lapse image series were acquired of tumorspheres to visualize attachment to Geltrex and migration for the first 24 hours (see Supplementary Video 1). The timelapse gave us an idea of how the tumorspheres transition from a free-floating state to becoming attached to the Geltrex (illustrated in Figure S3A). To examine the intercellular heterogeneity within the tumorspheres, tumorspheres were seeded in wells containing a Geltrex-coated coverslips and incubated those overnight for subsequent immunostaining and high-resolution fluorescence microscopy. In the first instance, smaller tumorspheres were stained for nestin expression to allow for high-resolution imaging of whole tumorspheres. On visual inspection, the lower slices of the microscopy Z-stack, showed that the tumorsphere core was nestin-negative, contrary to the positive nestin staining in the periphery (Figure S3B). This pattern of nestin expression in the cells prompted us to look for more differentiated cells in the tumorspheres. This was done by co-staining for GFAP and nestin/vimentin. Nestin and vimentin are known to associate with invasive cancer cells and cancer stem-like cells in GBM, whereas GFAP expression was indicative of a less invasive phenotype and is expressed in opposition to nestin, perhaps allowing for a phenotypical distinction [18-21]. Images obtained in the periphery of tumorspheres showed cells highly positive for vimentin and nestin and less positive for GFAP (Figure 1A, Figure S4). Interestingly, long projections were shown to stretch from the core of tumorspheres towards the periphery, possibly resembling astrocytic end-feet or tumor microtubes [22].

\section{***INSERT FIGURE $1 * * *$}

To further illustrate the phenotypical gradient from the core to the periphery, images were taken close to the core with an overlapping image towards the periphery. Here, less nestin-positive and more GFAPpositive cells were observed by the core (Figure 1B), whereas the peripheral cells were all nestin and GFAPpositive. However, despite being present, the GFAP displayed a fragmented (or non-filamentous) structure in the periphery, which might indicate an ongoing degradation of GFAP at the time of acquisition (less than 
24 hours after seeding the tumorspheres) (Figure 1C, Figure S5). Close to the core, GFAP expression was predominantly displayed as filamentous structures (Figure 1B-C, Figure S5). Orthogonal views showed a double-layer of cells close to the core with the top cell-layer mainly being nestin-positive/GFAP-negative and the bottom layer mainly nestin-negative/GFAP-positive. In the tumorsphere periphery, orthogonal views confirm these observations of fragmented GFAP expression, which also appeared to localize inside the nucleus (Figure 1C, Figure S5). Quantification of the GFAP and nestin expression revealed significant differences in the GFAP-to-nestin ratios when comparing the core and peripheral regions of the tumorspheres (Figure 1D). This underscored the observation that GFAP expression is decreased with the increase in migratory capacity of the tumorsphere cells.

To examine whether a heterogenous expression of GFAP/nestin was also evident in whole non-adherent tumorspheres (free-floating), we fixed and paraffin-embedded whole tumorspheres and cut them in $4 \mu \mathrm{m}$ sections for immunofluorescence staining (Figure 2A). Most cells in the tumorspheres were nestin-positive with the nestin staining covering $66 \%$ of the tumorspheres, and only $10 \%$ appearing to be GFAP-positive (Figure 2B). The GFAP pattern appeared quite diffuse, but the cells in the outermost periphery were GFAPnegative and nestin-positive, confirming the heterogenous gradient shown in the Geltrex setup.

\section{***INSERT FIGURE $2 * * *$}

\section{Tumorsphere phenotypic gradient is reflected in vivo}

Given the observations and phenotypic distinctions in the in vitro tumorsphere migration model, an in vivo experiment was set up using the same GBM tumorsphere culture to see if the in vitro model recapitulated the situation observed in vivo. Dissociated T78 GBM tumorspheres were stereotactically implanted into the striatum of nude mice and tumor growth monitored weekly with MRI (Figure S1). When the tumor reached a sufficient size $\left(10-20 \mathrm{~mm}^{3}\right)$, mice were sacrificed and whole brains were removed and stained for human GFAP and human nestin (Figure 3). Fluorescence slide scans of whole brain slices were correlated to the last MRI sequence obtained just before the mice were sacrificed and showed that fluorescence imaging was 
done approximately in the center of the tumor (Figure 3A-B, Figure S1C). Confocal microscopy of the same slides showed that the tumor core contained both nestin and GFAP-positive cells, and the tumor periphery showed a change towards nestin-positive and GFAP-negative cells with increased distance from the tumor core (Figure 3C-D). In the area between the more distant tumor cells and the tumor core, tumor cells positive for both nestin and GFAP were observed (Figure 3D). This could both indicate a transition zone towards a more nestin-positive phenotype or that the cells expressing both intermediate filaments possess migratory potential. The most distant tumor cells identified had migrated to the frontal superficial hippocampal formation and were nestin-positive and GFAP-negative (Figure 3E).

\section{***INSERT FIGURE $3 * * *$}

\section{Oxaliplatin reduces primary GBM tumorsphere migration in vitro}

After having established that the intratumoral heterogeneity was recapitulated in our in vitro model, we next wanted to study the potential of using the model as an assay of therapeutic efficacy. For this purpose, we utilized the platinum-based chemotherapeutic drug, oxaliplatin [23]. Single GBM tumorspheres were seeded onto Geltrex matrix on day 0 , and treatment groups were assigned on day 1 , followed by the addition of $5 \mu \mathrm{M}$ oxaliplatin. Phase-contrast images were acquired daily and the area of migration was measured (Figure 4A). On day 1, total area between groups was similar, however, a large reduction in migration was observed the following days after oxaliplatin treatment compared to controls (Figure 4B). During the experiment, the tumorspheres in the control group increased five-fold in size whereas the tumorspheres that received oxaliplatin increased only two-fold in size (Figure 4C). This indicated that the treatment had reduced the growth more than two-fold compared to that of the control after a single dose of oxaliplatin. When encapsulating oxaliplatin into stealth liposomes with low capacity for associating and endocytosing into the cells due to their polymer surface coating, the effects of oxaliplatin were markedly reduced (Figure 4C). Thus, the growth-inhibiting effects of oxaliplatin were successfully modelled and could be diminished by interfering with the interaction potential between the drug and GBM cells. 
304

\section{GBM-derived EVs stimulate GBM tumorsphere growth in vitro}

To illustrate that the Geltrex migration model can also be used to evaluate potential stimulatory effects on GBM cell migration, EVs isolated from a GBM-derived secondary cell line were applied to the system. The EVs were characterized by NTA and immunogold TEM, and subsequently added to the tumorspheres on day 1. The administrated EVs ranged in size from $\sim 50-350 \mathrm{~nm}$ with most of the EVs being around $150 \mathrm{~nm}$ (Figure 5A). NTA measurements on EV CTRL (EVs isolated from non-conditioned medium) did not yield enough events for analysis, thus were regarded as being EV-depleted. The tetraspanin proteins CD9, CD63 and CD81 are among the most widely used EV markers, and to validate that the isolated EVs used in this study were in fact EVs, we performed immunogold staining with a cocktail of monoclonal antibodies against these three types of tetraspanins followed by morphological assessment using TEM. The antibody-gold nanoparticle complexes showed an association to the outer membrane of the particles, indicating that the particles were positive for one or more of the tetraspanins and thus confirming that they were EVs (Figure $5 B$, Figure S6). The tumorspheres that received GBM EVs showed a significant increase in area compared to all the controls (Figure 5C-D). The GBM EVs increased GBM cell migration by more than $30 \%$ compared to both NTC and EV CTRL (EVs isolated from non-conditioned medium). TGF- $\beta 1$ was included as a simple positive control but did not enhance the migration of the cells in our setup. These results indicate that EVs isolated from a secondary GBM cell line could significantly stimulate GBM tumorsphere migration in vitro. Thus, it was demonstrated that both stimulatory and inhibitory effects on GBM cell migration could be measured using this model.

***INSERT FIGURE 5***

\section{DISCUSSION}


The highly invasive behavior of GBM limits therapeutic efficacy of current treatment strategies, which is substantiated by the almost ubiquitous occurrence of relapse [24]. Only subtle progress in patient prognosis has been made during the past two decades with a two-month increase in median survival by the addition of temozolomide to the treatment regimen [25]. The cells that most frequently invade the surrounding brain parenchyma and migrate far away from the tumor core (or primary tumor) have been shown to possess stem-like properties [26-28]. Understanding the invading and migrating GBM cells potentially harbors an avenue for improving treatment and therefore patient prognosis. Here, we presented a quantitative migration model based on GBM tumorspheres for assessment of cancer inhibiting or stimulating substances.

Generally, the study of GBM invasion and migration dynamics and the effects of different treatments on this property in vitro is limited by the model cell line and the assay of choice. Many different quantitative cell invasion and migration assays exist, including the wound-healing assay, transwell assay, cell exclusion assay, and fence (or ring) assay [4]. One feature is common for these assays; the cells are often conveniently grown as an adherent cell monolayer, typically with the addition of serum to the culture medium. Tumorspheres on the other hand are grown in absence of serum and preferentially in stem cellpromoting medium, which can induce and maintain a cellular heterogeneity within tumorspheres [29]. Tumorspheres can be generated from established cell cultures that are usually grown as a monolayer after a period of weaning or from primary cell lines directly isolated from tumor tissue $[30,31]$. The drawback of using monolayer cells in such an assay is that the cells might already have gone through a harsh selection process immediately after isolation, i.e. the selection of mesenchymal-like cells based on adherence, and might therefore not represent intercellular heterogeneity as well as tumorspheres from primary cells would do [29]. For example, drug resistance is different in cells grown either in 2D or 3D cultures, where the 3Dcultured cells appeared to be more resistant in the study by Imamura et al. [30]. Here, they used adherent cells as a 2D culture and induced non-adherent tumorspheres from the same cells to produce a 3D culture, which indicates that the 3D organization of the cells could play a role in drug response [30]. In this study, 
we presented a GBM tumorsphere migration model using primary GBM cells isolated under tumorsphereinducing conditions. We used the Matrigel-derived ECM Geltrex as the migration matrix of choice due to its complex composition, the fact that it is hESC-qualified and has a reduced concentration of growth factors.

The cellular heterogeneity in our tumorsphere model showed a crude differential phenotypic gradient of cells based on their location in the tumorsphere. Nestin and vimentin were found to be highly expressed in the tumorsphere periphery, whereas GFAP was expressed both in the core and periphery. However, the structure of GFAP in the periphery appeared fragmented, which could indicate an ongoing degradation of GFAP and hence a cellular phenotype shift from GFAP-positive towards nestin/vimentinpositive [32]. This reduction in GFAP expression was also reflected, when quantitatively comparing the core and peripheral regions of the tumor. We further showed a similar distribution of nestin/GFAP staining in free-floating tumorspheres in vitro and in vivo in a mouse intracranial xenograft setup using the same primary GBM cells. Nestin has for a couple of decades been known as a multi-lineage progenitor marker and was in embryonic stem cells shown to be expressed in the progenitor 'transition' period and then turned off when cells fully differentiated $[33,34]$. Similarly, glial progenitor cells were nestin-positive and GFAP-negative, but at the end of cellular differentiation, GFAP had replaced nestin [35]. Nestin has further been associated with a migratory phenotype, where it facilitates migration of neural stem cells and directs inflammatory cell migration in atherosclerosis [36,37]. In cancer, nestin expression is generally associated with cancer stem-like cells, and more specifically in GBM, nestin has been shown to be useful for identifying migrating tumor cells [38,39]. Downregulation of nestin demonstrated a reduction of tumorsphere formation and tumor size in vivo, and overexpression results in increased cell growth, tumorsphere formation and cell invasion [40]. However, the opposite has also been reported, where downregulation of nestin increased matrix degradation and pFAK localization to focal adhesions for increased prostate cancer cell invasion, which could indicate functional differences between different types of cancer [41]. In the case of human GBM tumors, nestin is expressed in the tumor periphery and in the invading tumor cells [42]. Munthe et al. reported nestin-positive cells both in the core and periphery of 
human GBM tumors, and showed that the same distribution could be seen in a GBM xenograft model, using the same cells $(\mathrm{T} 78)$ as in our study $[27,28]$. This could indicate that our in vitro tumorsphere migration model shows a crude similarity to human GBM tumors, thus demonstrating a biological relevance for our tumorsphere model in drug screening and cellular responses to the drugs used.

We demonstrated that oxaliplatin could reduce tumorsphere migration by more than twofold, and by encapsulating oxaliplatin in stealth liposome these therapeutic effects were reduced. The cell repulsion effects of stealth liposome formulations can thus be reliably assessed in this model even after a period of four days as shown here, which could indicate that this migration model could provide a useful tool for researchers working on various drug delivery systems [43]. The model could also be used to visualize stimulation of GBM tumorsphere migration by adding EVs harvested from a GBM cancer cell line. The EV field is rapidly expanding with thousands of new publications each year ranging from basic biology to drug delivery. Models, such as the one presented here, could potentially contribute to elucidating functional effects of both engineered EVs for drug delivery and specific biological populations of EVs, since several studies have shown that parts of the functional cell-cell communication in GBM happens via EVs [44-47]. In addition to the quantitative assessment of EVs, the EVs secreted from the cells in such a setup can be isolated and analyzed with a potential minimum of serum-derived contaminants as they are grown under serum-free conditions, which might help with overcoming a technical barrier in EV analyses [48].

\section{METHODOLOGICAL CONSIDERATIONS}

Although we do not directly demonstrate a high-throughput model, a few protocol alterations could easily make it high-throughput for GBM drug screening. We used 24-well plates and manually picked single tumorspheres and seeded into the wells, but this process could be replaced by a limiting dilution of tumorspheres into 96-well plates. We manually acquired images of the tumorspheres and this could be optimized by acquiring an automated image station such as IncuCyte (Essen Bioscience) or Celigo (Nexcelom Bioscience). Vinci et al. demonstrated a high-throughput 3D GBM tumorsphere invasion assay 
using such an image station with automated quantitation of invasion [49]. To further enhance cellular complexity of our model, GBM organoids could be used. Development in the field of organoid research is accelerating and several techniques and models within the GBM field are emerging, showing much more cellular complexity than tumorspheres [50,51]. However, generation of organoids takes longer time (up to several months) and thus serves as rate-limiting for the use in high-throughput drug screens [50]. In between the convenience of monolayer cultures and the lengthy process of organoid generation, tumorspheres might present an acceptable middle ground with both convenient culturing and sufficient complexity.

\section{CONCLUSIONS}

In conclusion, we presented a GBM tumorsphere migration model with intercellular heterogeneity, which might provide a relevant in vitro model for drug response evaluation. The cellular organization and complexity of cancers are hard to reproduce in vitro for high-throughput drug screening and drug response evaluation, but we believe that tumorsphere migration models such as presented here could be an important step towards more accurate drug screening prior to evaluation in expensive pre-clinical animal models. The research in this field is fortunately accelerating with more advanced cell models and equipment for better analysis. 
420

421

422

423

\section{ACKNOWLEDGEMENTS}

The authors would like to thank Leonid Gurevich from the Department of Physics and Nanotechnology, Aalborg University for providing access to the Nanosight LM10 to estimate EV size and concentrations. We would also like to thank Professor Torben Moos and Professor Vladimir Zachar from the Department of Biomedicine, Aalborg University for providing access to their respective microscopy facilities. The Core Facility for Integrated Microscopy (CFIM, University of Copenhagen) are acknowledged for providing the facilities and expertise to perform confocal microscopy and fluorescence slide scanning. This study is supported by Augustinus Fonden (Grant no. 15-5052).

\section{CONFLICT OF INTEREST}

The authors declare no conflict of interest.

\section{SUPPLEMENTARY INFORMATION}

Supplementary information is available at the journal's website.

\section{REFERENCES}

1. Bleeker FE, Molenaar RJ, Leenstra S. Recent advances in the molecular understanding of glioblastoma. J Neurooncol. 2012;108(1):11-27.

2. Alifieris C, Trafalis DT. Glioblastoma multiforme: Pathogenesis and treatment. Pharmacol Ther. 2015;152:63-82.

3. Lathia JD, Heddleston JM, Venere M, Rich JN. Deadly teamwork: neural cancer stem cells and the tumor microenvironment. Cell Stem Cell. 2011;8(5):482-5.

4. Kramer N, Walzl A, Unger C, Rosner M, Krupitza G, Hengstschläger M, et al. In vitro cell migration and invasion assays. Mutat Res Mutat Res. 2013;752(1):10-24. 
$441 \quad 5 . \quad$ Weiswald L-B, Bellet D, Dangles-Marie V. Spherical Cancer Models in Tumor Biology. Neoplasia. 2015;17(1):1-15.

6. Kang S-G, Cheong J-H, Huh YM, Kim EH, Kim SH, Chang JH. Potential use of glioblastoma tumorsphere: clinical credentialing. Arch Pharm Res. 2015;38(3):402-7.

7. Li S, Li Q. Cancer stem cells and tumor metastasis. Int J Oncol. 2014;44(6):1806.

8. Shiozawa Y, Nie B, Pienta KJ, Morgan TM, Taichman RS. Cancer stem cells and their role in metastasis. Pharmacol Ther. 2013;138(2):285-93.

9. Santo VE, Rebelo SP, Estrada MF, Alves PM, Boghaert E, Brito C. Drug screening in 3D in vitro tumor models: overcoming current pitfalls of efficacy read-outs. Biotechnol J. 2017;12(1):1600505.

10. Sodek KL, Brown TJ, Ringuette MJ. Collagen I but not Matrigel matrices provide an MMP-dependent barrier to ovarian cancer cell penetration. BMC Cancer. 2008;8:223.

11. Irimia D. Cell Migration in Confined Environments. In: Methods in cell biology. 2014 [cited 2018]. p. $141-53$.

12. Banyard J, Chung I, Migliozzi M, Phan DT, Wilson AM, Zetter BR, et al. Identification of genes regulating migration and invasion using a new model of metastatic prostate cancer. BMC Cancer.

13. Jensen SS, Meyer M, Petterson SA, Halle B, Rosager AM, Aaberg-Jessen C, et al. Establishment and Characterization of a Tumor Stem Cell-Based Glioblastoma Invasion Model. Ulasov I, editor. PLoS One. 2016;11(7):e0159746.

14. Jensen SS, Aaberg-Jessen C, Andersen C, Schrøder HD, Kristensen BW. Glioma Spheroids Obtained via Ultrasonic Aspiration Are Viable and Express Stem Cell Markers. Neurosurgery. 2013;73(5):86886. 
15. Benzina S, Debomy F, Bergerat J-P, Denis J-M, Gueulette J, Dufour P, et al. The cytotoxicity of highlinear energy transfer radiation is reinforced by oxaliplatin in human glioblastoma cells. Cancer Lett. 2007;254(1):54-62.

16. Schindelin J, Arganda-Carreras I, Frise E, Kaynig V, Longair M, Pietzsch T, et al. Fiji: an open-source platform for biological-image analysis. Nat Methods. 2012;9(7):676-82.

17. Johnsen KB, Bak M, Melander F, Thomsen MS, Burkhart A, Kempen PJ, et al. Modulating the antibody density changes the uptake and transport at the blood-brain barrier of both transferrin receptor-targeted gold nanoparticles and liposomal cargo. J Control Release. 2019;295:237-49.

18. Christensen K, Schrøder HD, Kristensen BW. CD133+ niches and single cells in glioblastoma have different phenotypes. J Neurooncol. 2011;104(1):129-43.

19. Johannessen T-CA, Wang J, Skaftnesmo K-O, Sakariassen $P \varnothing$, Enger $P \varnothing$, Petersen K, et al. Highly infiltrative brain tumours show reduced chemosensitivity associated with a stem cell-like phenotype. Neuropathol Appl Neurobiol. 2008;35(4):no-no.

20. Rutka JT, Ivanchuk S, Mondal S, Taylor M, Sakai K, Dirks P, et al. Co-expression of nestin and vimentin intermediate filaments in invasive human astrocytoma cells. Int J Dev Neurosci. 17(56):503-15.

21. Kitai R, Horita R, Sato K, Yoshida K, Arishima H, Higashino Y, et al. Nestin expression in astrocytic tumors delineates tumor infiltration. Brain Tumor Pathol. 2010;27(1):17-21.

22. Osswald M, Jung E, Sahm F, Solecki G, Venkataramani V, Horstmann H, et al. Brain tumor cells interconnect to a functional and resistant network. Nature, rev. 2015;

23. Bruno PM, Liu Y, Park GY, Murai J, Koch CE, Eisen TJ, et al. A subset of platinum-containing chemotherapeutic agents kills cells by inducing ribosome biogenesis stress. Nat Med. 
485

2017;23(4):461-71.

24. Ostrom QT, Gittleman H, Xu J, Kromer C, Wolinsky Y, Kruchko C, et al. CBTRUS Statistical Report: Primary Brain and Other Central Nervous System Tumors Diagnosed in the United States in 20092013. Neuro Oncol. 2016;18(suppl_5):v1-75.

25. Stupp R, Mason WP, van den Bent MJ, Weller M, Fisher B, Taphoorn MJB, et al. Radiotherapy plus concomitant and adjuvant temozolomide for glioblastoma. N Engl J Med. 2005;352(10):987-96.

26. Kalkan R. Glioblastoma Stem Cells as a New Therapeutic Target for Glioblastoma. Clin Med Insights Oncol. 2015;9:95-103.

27. Munthe S, Petterson SA, Dahlrot RH, Poulsen FR, Hansen S, Kristensen BW. Glioma Cells in the Tumor Periphery Have a Stem Cell Phenotype. Castro MG, editor. PLoS One. 2016;11(5):e0155106.

28. Munthe S, Sørensen MD, Thomassen M, Burton M, Kruse TA, Lathia JD, et al. Migrating glioma cells express stem cell markers and give rise to new tumors upon xenografting. J Neurooncol. 2016;130(1):53-62.

29. Günther HS, Schmidt NO, Phillips HS, Kemming D, Kharbanda S, Soriano R, et al. Glioblastomaderived stem cell-enriched cultures form distinct subgroups according to molecular and phenotypic criteria. Oncogene. 2008;27(20):2897-909.

30. Imamura Y, Mukohara T, Shimono Y, Funakoshi Y, Chayahara N, Toyoda M, et al. Comparison of 2Dand 3D-culture models as drug-testing platforms in breast cancer. Oncol Rep. 2015;33(4):1837-43.

31. Zanoni M, Piccinini F, Arienti C, Zamagni A, Santi S, Polico R, et al. 3D tumor spheroid models for in vitro therapeutic screening: a systematic approach to enhance the biological relevance of data obtained. Sci Rep. 2016;6(1):19103.

32. Zhou R, Skalli O. TGF- $\alpha$ Differentially Regulates GFAP, Vimentin, and Nestin Gene Expression in U- 

373 MG Glioblastoma Cells: Correlation with Cell Shape and Motility. Exp Cell Res. 2000;254(2):26978.

33. Wiese C, Rolletschek A, Kania G, Blyszczuk P, Tarasov K V., Tarasova Y, et al. Nestin expression ? a property of multi-lineage progenitor cells? Cell Mol Life Sci. 2004;61(19-20):2510-22.

34. Messam CA, Hou J, Major EO. Coexpression of Nestin in Neural and Glial Cells in the Developing Human CNS Defined by a Human-Specific Anti-nestin Antibody. Exp Neurol. 2000;161(2):585-96.

35. Fraichard A, Chassande O, Bilbaut G, Dehay C, Savatier P, Samarut J. In vitro differentiation of embryonic stem cells into glial cells and functional neurons. J Cell Sci. 1995;108 ( Pt 10):3181-8.

36. Yan S, Li P, Wang Y, Yu W, Qin A, Liu M, et al. Nestin regulates neural stem cell migration via controlling the cell contractility. Int J Biochem Cell Biol. 2016;78:349-60.

37. del Toro R, Chèvre R, Rodríguez C, Ordóñez A, Martínez-González J, Andrés V, et al. Nestin+ cells direct inflammatory cell migration in atherosclerosis. Nat Commun. 2016;7:12706.

38. Neradil J, Veselska R. Nestin as a marker of cancer stem cells. Cancer Sci. 2015;106(7):803-11.

39. Kitai R, Horita R, Sato K, Yoshida K, Arishima H, Higashino Y, et al. Nestin expression in astrocytic tumors delineates tumor infiltration. Brain Tumor Pathol. 2010;27(1):17-21.

40. Matsuda Y, Ishiwata T, Yoshimura H, Hagio M, Arai T. Inhibition of nestin suppresses stem cell phenotype of glioblastomas through the alteration of post-translational modification of heat shock protein HSPA8/HSC71. Cancer Lett. 2015;357(2):602-11.

41. Hyder CL, Lazaro G, Pylvänäinen JW, Roberts MWG, Qvarnström SM, Eriksson JE. Nestin regulates prostate cancer cell invasion by influencing the localisation and functions of FAK and integrins. J Cell Sci. 2014;127(Pt 10):2161-73. 
42. Ylivinkka I, Sihto H, Tynninen O, Hu Y, Laakso A, Kivisaari R, et al. Motility of glioblastoma cells is driven by netrin-1 induced gain of stemness. J Exp Clin Cancer Res. 2017;36(1):9.

43. Bozzuto G, Molinari A. Liposomes as nanomedical devices. Int J Nanomedicine. 2015;10:975-99.

44. Johnsen KB, Gudbergsson JM, Skov MN, Pilgaard L, Moos T, Duroux M. A comprehensive overview of exosomes as drug delivery vehicles - Endogenous nanocarriers for targeted cancer therapy. Biochim Biophys Acta. 2014;1846(1):75-87.

45. Belting M, Christianson HC. Role of exosomes and microvesicles in hypoxia-associated tumour development and cardiovascular disease. J Intern Med. 2015;278:251-63.

46. van der Vos KE, Abels ER, Zhang X, Lai C, Carrizosa E, Oakley D, et al. Directly visualized glioblastomaderived extracellular vesicles transfer RNA to microglia/macrophages in the brain. Neuro Oncol. 2015;(February):nov244.

47. Skog J, Würdinger T, van Rijn S, Meijer DH, Gainche L, Sena-Esteves M, et al. Glioblastoma microvesicles transport RNA and proteins that promote tumour growth and provide diagnostic biomarkers. Nat Cell Biol. 2008;10(12):1470-6.

48. Gudbergsson JM, Johnsen KB, Skov MN, Duroux M. Systematic review of factors influencing extracellular vesicle yield from cell cultures. Cytotechnology. 2015;

49. Vinci M, Box C, Eccles SA. Three-dimensional (3D) tumor spheroid invasion assay. J Vis Exp. 2015;(99):e52686.

50. Hubert CG, Rivera M, Spangler LC, Wu Q, Mack SC, Prager BC, et al. A Three-Dimensional Organoid Culture System Derived from Human Glioblastomas Recapitulates the Hypoxic Gradients and Cancer Stem Cell Heterogeneity of Tumors Found In Vivo. Cancer Res. 2016;76(8):2465-77.

51. da Silva B, Mathew RK, Polson ES, Williams J, Wurdak H. Spontaneous Glioblastoma Spheroid 

$R \& D .2018 ; 247255521876462$.

552

553 
Figure 1: Intra-tumorsphere cellular heterogeneity. (A) Immunofluorescence stainings for nestin or Vimentin (green) and GFAP (red). Images show nestin and Vimentin expression most prominent in the tumorsphere periphery and GFAP expression mostly from the tumorsphere core with GFAP-positive filaments stretching from core to periphery (arrows). Scale bars: $20 \mu \mathrm{m}$. (B) Overlapping immunofluorescence images acquired to visualize differences in nestin/GFAP expression based on cellular location. The periphery shows filamentous nestin distribution and non-filamentous (or fragmented) GFAP distribution, which was also seen within the nucleus. Closer to the core, where a double cell layer was observed, the bottom cells appeared GFAP-positive/nestin-negative and the top cells appeared nestinpositive/GFAP-negative. Yellow stippled line approximately indicates the transition zone. Arrows indicate examples of filmentous GFAP. Scale bars: $20 \mu \mathrm{m}$. (C) Zooms on orthogonal regions from both periphery and core, which shows fragmented and intra-nuclear GFAP localization in the periphery and filamentous cytosolic GFAP in the core. In the periphery, arrows indicate fragmented GFAP inside nuclei and in the core, arrows show nuclei free of GFAP. (D) Quantification of GFAP:Nestin ratio between tumorsphere core and periphery. Data is presented as Mean + SD from four separate images.

Figure 2: Nestin/GFAP distribution in free-floating tumorspheres. (A) Immunofluorescence of nestin (green) and GFAP (red) showed most of the cells being nestin-positive and fewer cells GFAP-positive. Scale bar: 50 $\mu \mathrm{m}$ (B) Estimation of area coverage for each signal in percent using threshold analysis. Total nestin coverage was around $66 \%$ and GFAP total coverage around $10 \%$.

Figure 3: Distribution of nestin/GFAP in a GBM mouse xenograft model using the same cells. (A) Fluorescence slide scanning of whole brain slices stained with GFAP (green) and nestin (red). Image show both nestin and GFAP expression in the tumor core, but peripheral cells appear only nestin-positive (see asterix). Scale bar: $1 \mathrm{~mm}$. (B) MRI of mouse brain showing the tumor just prior to sacrificing the mouse. Image shows that the fluorescent stainings were done on sections from the middle of the tumor (more 
details in Figure S1C). (C) Fluorescence laser-scanning confocal image of from the tumor core showing both nestin and GFAP-positive cells. Scale bar: $50 \mu \mathrm{m}$. (D) Fluorescence laser-scanning confocal image of the tumor periphery showing the tumor cells farthest from the tumor being nestin-positive and GFAP-negative. Scale bar: $50 \mu \mathrm{m}$. (E) Fluorescence slide scan zoom-in on frontal superior hippocampal formation showing nestin-positive/GFAP-negative tumor cells. Scale bar: $200 \mu \mathrm{m}$.

Figure 4: Inhibition of tumorsphere migration with Oxaliplatin. (A) Daily phase-contrast images of representative tumorspheres from each group visualizing the difference in area of migration after treatment initiated on D1. Scale bar: $400 \mu \mathrm{m}$. (B) Bar chart of total tumorsphere area measured on each day. (C) Tumorsphere migration normalized by applying a fold-change from each day after treatment (D2D4) to the day of treatment (D1). Normalized data is presented as mean \pm SEM. $P=0.03-0.05$ on D3-D1, $P$ $=0.002$ on D4-D1.

Figure 5: Stimulation of tumorsphere migration with GBM-derived extracellular vesicles. (A) Size distribution of EVs measured with NTA. (B) characterization of EVs by immunogold TEM using a cocktail of antibodies against the tetraspanins CD9, CD63 and CD81. Scale bar: $100 \mathrm{~nm}$. (C) Bar chart of total tumorsphere area measured on each day. (D) Tumorsphere migration normalized by applying a foldchange from each day after treatment (D2-D4) to the day of treatment (D1). EV CTRL consisted of EVs isolated from non-conditioned medium and TGF- $\beta 1$ was included as a positive migration control, however, it did not induce any significant effects. Normalized data is presented as mean \pm SEM. $P=0.002-0.02$ on D3-D1, $P=0.002-0.02$ on D4-D1. 

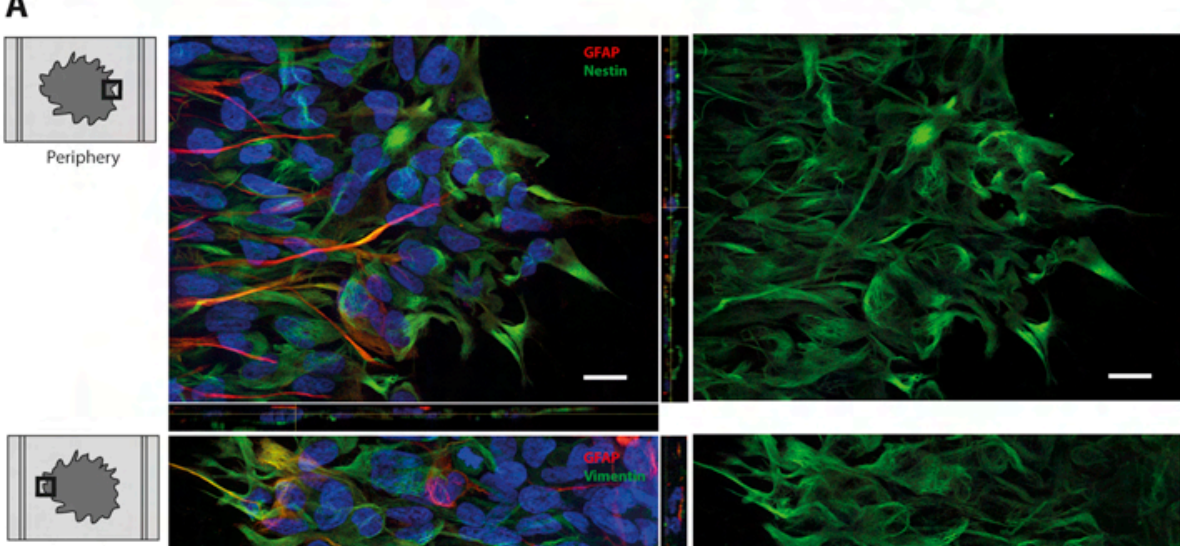

Periphery
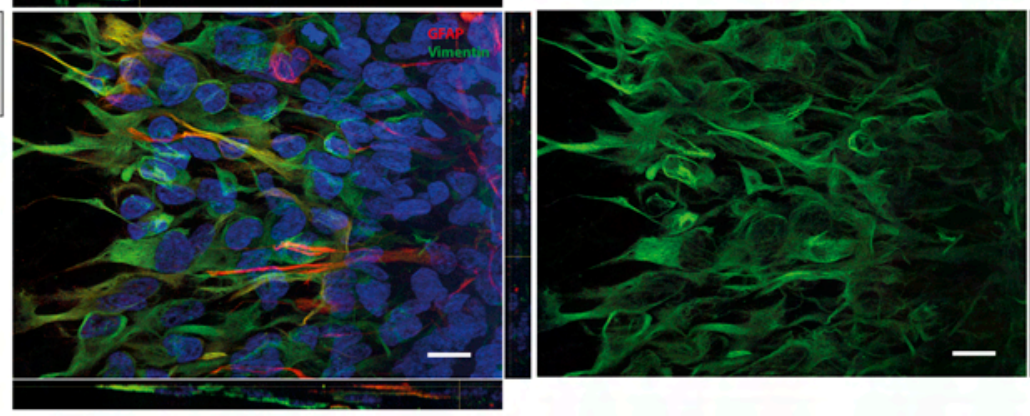

$\downarrow$

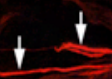

B

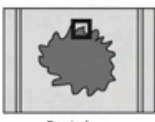

Periphery

C

Periphery

t t

$+t$

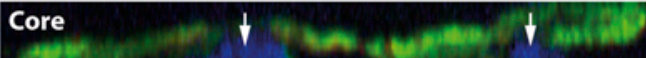
4 $\downarrow$ 
A

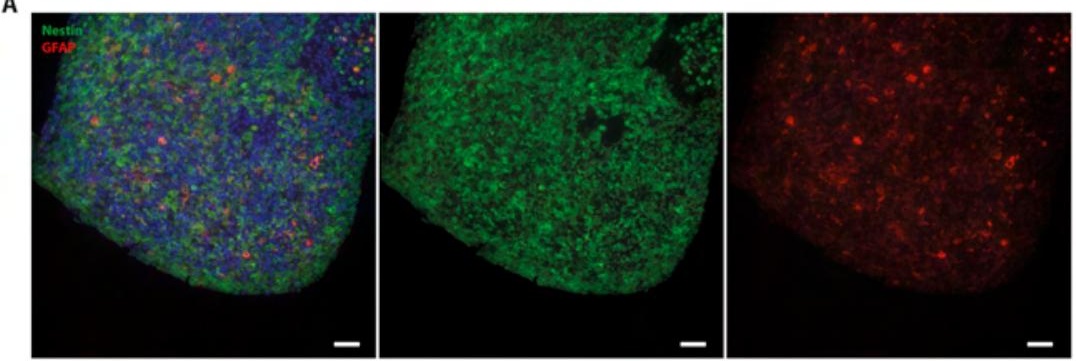

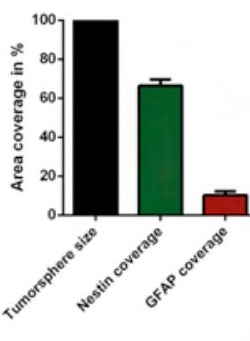




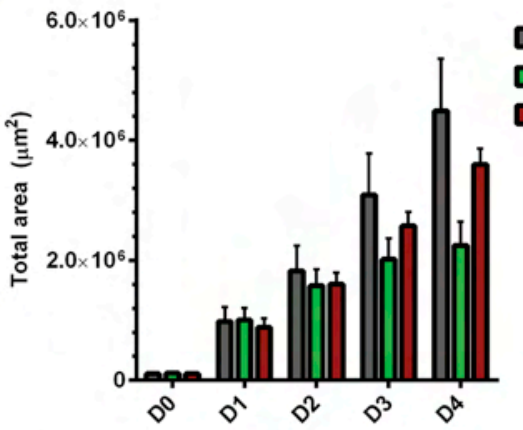

Day 0
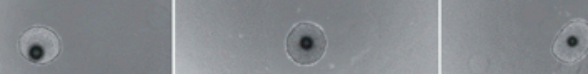

C

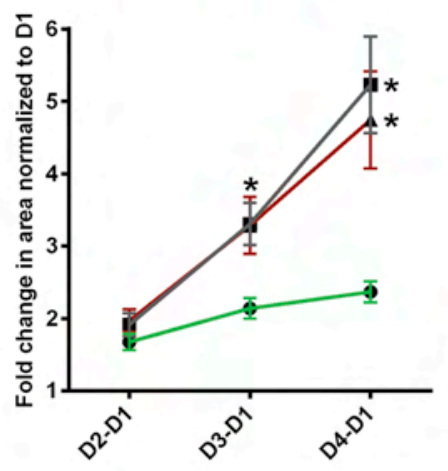




\section{HIGHLIGHTS}

- Intratumoral heterogeneity is present in complex primary GBM tumorspheres in vitro

- Heterogeneity is visualized as a function of migration by differential distribution of nestin/vimentin and GFAP between core and periphery in vitro and in vivo

- Patient-derived GBM tumorspheres are promising for use in drug screens and studies of GBM biology in vitro and in vivo 